\title{
Intradialytic exercise improves physical function and reduces intradialytic hypotension and depression in hemodialysis patients
}

So Yon Rhee ${ }^{1}$, Jin Kyung Song ${ }^{1}$, Suk Chul Hong ${ }^{1}$, Jae Won Choi ${ }^{1}$, Hee Jung Jeon ${ }^{1}$, Dong Ho Shin ${ }^{1}$, Eun Hee $\mathrm{Ji}^{2}$, Eun-Hee Choi ${ }^{3}$, Jiyeon $\mathrm{Lee}^{4}$, Aram Kim ${ }^{4}$, Seung Wook $\mathrm{Choi}^{4}$, and Jieun $\mathrm{Oh}^{1}$

Departments of ${ }^{1}$ Internal Medicine and ${ }^{2}$ Psychiatry, Hallym University Kangdong Sacred Heart Hospital, Seoul; ${ }^{3}$ Department of Rehabilitation Medicine, Hallym University Chuncheon Sacred Heart Hospital, Chuncheon; ${ }^{4}$ Department of Sports and Leisure, Sungshin University, Seoul, Korea
Received: January 16, 2017

Revised : February 27, 2017

Accepted: April 2, 2017

\section{Correspondence to}

Jieun Oh, M.D.

Department of Internal Medicine, Hallym University Kangdong Sacred Heart Hospital, 150 Seongan-ro, Gangdong-gu, Seoul 05355, Korea

Tel: +82-2-2224-2190

Fax: +82-2-478-6925

E-mail:jieunmd@gmail.com
Background/Aims: As numbers of maintenance hemodialysis patients are growing, debilitating conditions of muscle wasting and atrophy are becoming some of the greatest concerns in end-stage renal disease patients. Exercise training has various potential benefits in terms of prevention of a sustained decline in functional status. This study aimed to evaluate the physical, psychological, laboratory, and dialysis-related effects of intradialytic exercise.

Methods: We enrolled 22 patients from a hemodialysis center for a 6-month nonrandomized prospective trial. Combination of aerobic exercise with bicycle ergometer and anaerobic exercise with elastic bands was conducted during hemodialysis. Data including physical fitness test results, dialysis-related measurements, and biochemical laboratory results were collected at baseline, 3, and 6 months. Depression and quality of life were assessed using Beck Depression Inventory and Short Form-36 health survey.

Results: After exercise completion, there were significant improvements in back muscle power, forward and backward trunk flexibility, vertical jump, elbow flexion, sit to stand test, and 6 -minute walk test $(p<0.05)$. No significant changes were observed in dry weight, blood pressure, $\mathrm{Kt} / \mathrm{V}$, and biochemical variables, except for intradialytic hypotension $(p<0.05)$. For depression, Beck Depression Inventory showed statistically significant enhancement $(p<0.05)$. Scores of Short Form-36 health survey did not show significant increase in each domain, except for bodily pain $(p<0.05)$.

Conclusions: Combined aerobic and anaerobic exercise training during dialysis was found to be effective on physical health status, intradialytic hypotension, and depression in terms of mental health. Therefore, the findings of the current study may provide an appropriate guidance for encouraging exercise by nephrologists.

Keywords: Renal dialysis; Intradialytic exercise; Physical function; Intradialytic hypotension; Depression

\section{INTRODUCTION}

Exercise training is generally recommended to people even if they have comorbidities in order to improve their physical and psychological health. According to the data from the United States Renal Data System 
2015, overall prevalence of chronic kidney disease in the general population was approximately $14 \%$ and the great proportionate increase in the incidence of treated end-stage renal disease (ESRD) in the Republic of Korea (120\%) over the period between 2000/2001 and 2012/2013 were reported [1].

The importance of exercise for the maintenance hemodialysis (MHD) patients is emphasized in some guidelines. The National Kidney Foundation, using the Kidney Disease Outcome Quality Initiative guidelines on the management of cardiovascular disease in patients on MHD (2005), advises nephrologists to counsel and regularly encourage patients on MHD to increase levels of physical activity despite of numerous barriers [2]. European guidelines on nutrition in hemodialysis (HD) patients also evidently recommend regular physical exercise [3].

MHD patients are profoundly deconditioned, often vulnerable and older with muscle wasting and altered nutritional status. Dialysis leads to distinct metabolic changes such as hypovolemia, electrolyte imbalance, and systemic inflammation [4]. As a result, the exercise capacity in ESRD is about 50\% to $60 \%$ of that seen in normal subjects [5].

Exercise training has a myriad of potential benefits that can ameliorate a sustained decline in functional status. In ESRD patients, chronic regular exercise showed improved aerobic capacity, muscle strength, and cardiovascular function [6]. Moreover, exercise revealed strong positive correlations with dialysis efficacy, high-sensitivity C-reactive protein, blood pressure, arterial stiffness, health-related quality of life (QOL), and depression $[6,7]$. However, there might be several exercise related adverse effects which include fatigue, hypotensive episodes, musculoskeletal complications, and rare cardiovascular complications [7].

Toxic substances, also called uremic toxins, in the blood result in malaise, paraesthesia, mental impairment, peripheral circulatory disturbance, and muscle dysfunction [8]. While patients exercise during dialysis, those toxins move through the dialyzer with a large flux from the tissue to the vascular compartment. It results in increased muscle blood flow and opened capillary surface area [9]. Besides, the intradialytic exercise showed not only a better adoption and adherence but also a lower drop-out and greater compliance [7].
Commonly, exercises are divided into aerobic and anaerobic types. Aerobic exercise is usually prescribed to enhance endurance by using large muscle groups (e.g., cycle ergometers). On the other hand, anaerobic exercise is proven to increase muscle size and strength (e.g., elastic bands, dumbbells) [10]. Therefore, combined aerobic and anaerobic exercise should produce greater effects than each exercise method alone.

We hypothesized that there might be numerous positive improvements after the completion of 6-month intradialytic, combined aerobic and anaerobic exercise. Overall evaluation was thoroughly carried out to measure physical, psychological, and laboratory effects. In addition, we included patients who had episodes of intradialytic hypotension (IDH) unless they were unable to perform exercise. We planned to examine the effect of exercise on IDH that was previously impossible due to exclusion of IDH patients.

\section{METHODS}

\section{Patients}

The study was conducted in a single outpatient hemodialysis center at the Hallym University Kangdong Sacred Heart Hospital in Seoul, Republic of Korea. Twenty-two patients were initially enrolled for a non-randomized 6-month prospective trial of intradialytic exercise training. Three patients received MHD two times per week and the rest of them underwent dialysis three times a week. Participants included nine men and 13 women.

Patients were eligible for enrolment if they were adults (age $\geq 18$ years) on MHD for longer than 3 months with adequate dialysis therapy $(\mathrm{Kt} / \mathrm{V} \geq 1.2)$. Exclusion criteria included history of myocardial ischemia in the last 6 months, uncontrolled diabetes mellitus and hypertension, heart failure (New York Heart Association stage $\geq$ 3), symptomatic cardiovascular disease with functional disability, and unstable medical condition due to acute or chronic illness.

\section{Study design}

All physical tests and questionnaire on physical activity were completed by every patient before starting the exercise. From the beginning to the end, the exercise program was supervised and individualized by two physical 
educators who were doctoral students in physical education.

A 5-minute warm-up before exercise and a 5-minute cool-down following exercise were carried out each time. In addition, the exercise was performed in the first half of the dialysis session over a 6 months period. To measure exercise intensity subjectively, the Borg Rating of Perceived Exertion (RPE) scale ranging from 6 to 20 points was used. The exercise was started at 'fairly light' intensity (Borg RPE 11, 11\% to 60\% effort) aiming for 'some-what hard' intensity (Borg RPE 13 to 14, 13\% to $75 \%$ effort).

For aerobic exercise training, participants were trained to use stationary bicycle ergometer (Thera-fit Plus bed trainer, Medica Medizintechnik, Hochdorf, Germany) in a supine position for minimum of 30 minutes at each HD session. The speed, load, and duration were gradually increased with change of mode (automatic and passive) and application of sand bags. According to patients' performance, training loads were adjusted by trainers.

After cycling, patients performed exercise with elastic bands (Thera-Band, Hygenic, Alcron, MS, USA) with non-fistula containing arm in the supine position. Two to three sets of 10 to 15 repetitions of each exercise were conducted for approximately 5 to 10 minutes/day. To prevent slips and possible injuries, the band was double wrapped to a hand for a firm grasp. The anaerobic program included: elbow extension, elbow flexion, and side shoulder abduction. Anaerobic exercises were made progressively harder using different band colours (red, green, and blue) or band numbers (1 to 2) and increasing the number of repetitions.

Patients were frequently questioned whether they had any problems. In case of nausea, vomiting, chest pain, muscle pain or any other discomfort exercises were stopped. Patients were also permitted to stop and rest or train at a milder intensity.

\section{Data collection}

All outcome measures were collected at the baseline, month 3, and month 6. Baseline demographics and clinical information about participants were also obtained at the commencement of the intervention.

Physical fitness test

To assess physical ability, various tests and measure- ments were conducted. These are described below.

(1) Hand grip strength (HGS) was measured using mechanical dynamometer in the non-fistula hand.

(2) Back muscle power was measured using back muscle digital dynamometer.

(3) For elbow flexion test, the patient held a dumbbell in his or her hand for 30 seconds and then the number of elbow flexion was counted.

(4) The sit-to-stand test consisted of sitting down and standing as fast as possible for 60 seconds with arms held tightly against the chest.

(5) Flexibility assessment was carried out by using forward and backward trunk flexibility tests.

(6) Patients were asked to jump vertically with a line connected to a belt on his or her waist to check the length of ascending line.

(7) Arm and thigh circumferences were checked.

(8) A 6-minute walk test involved patients walking at the maximum speed in six minutes along a corridor next to the hemodialysis unit.

\section{Laboratory data}

Data related to dialysis and biochemical laboratory results were collected. Systolic blood pressure, diastolic blood pressure, and weight at the initiation of dialysis were recorded.

In this study, minimum intradialytic systolic blood pressure (< $90 \mathrm{mmHg}$, Nadir 90) or any hypotension related symptoms requiring nursing interventions were defined as IDH. The occurrence of IDH was checked in every dialysis session. The total numbers of IDH episodes were recorded separately at month $\circ$ (3 months prior to exercise), month 3 (first 3 months of exercise), and month 6 (last 3 months of exercise) time-points.

Dialysis efficacy was calculated using equation of single-pool Kt/V. All blood samples for examination of serum concentrations were drawn pre-dialysis. Parameters measured included white blood cell count, haemoglobin, haematocrit, platelet, intact parathyroid hormone, calcium, phosphate, uric acid, blood urea nitrogen, creatinine, glucose, cholesterol, high density lipoprotein, low density lipoprotein, triglyceride, protein, albumin, C-reactive protein, sodium, potassium, chloride, and total carbon dioxide. 


\section{Depression}

In order to assess the severity of symptoms and to diagnose depression, a direct face-to-face interview with a psychiatrist and Beck Depression Inventory (BDI) was used. For clinical diagnosis, it was categorized into three groups; no depression, mild depression and moderate depression. The BDI is composed of 21 items that can be self-rated and that include cognitive, emotional, motivational, and physical domains. A total score of o to 9 indicates the absence of depression, 10 to 15 indicates a mild depression, 16 to 23 indicates a moderate depression, and above 24 indicates a severe depression.

\section{Quality of life}

The self-reported QOL was evaluated using the Short Form-36 (SF36) items health survey. It includes eight independent subscales that can be calculated to assess physical and mental aspects of health. A total score can range from o to 100. A score of $\mathrm{o}$ is equivalent to maximum disability and a score of 100 is equivalent to no disability.

\section{Ethics statements}

Before conducting research, the study was reviewed and approved by Institutional Review Board of Hallym University Kangdong Sacred Heart Hospital (no. 07-28). The study protocol was abided by the ethical standards of the Declaration of Helsinki. Enough written and verbal information about the aim, procedure, predicted effects, and risks of the study was provided to all participants. If subjects agreed to participate in the trial then a written informed consent was signed.

\section{Statistical analysis}

Quantitative variables were expressed as mean \pm standard deviation. Repeated-measure of analysis of variance was used to figure out the difference of outcome measures in different time intervals. In all tests, $p$ values of less than 0.05 were considered statistically significant. All statistical analysis was performed using the IBM SPSS version 22.0 (IBM Corp., Armonk, NY, USA).

\section{RESULTS}

Baseline clinical characteristics of 22 eligible HD par- ticipants are described in Table 1. Patients ranged in age from 30 to 73 years with their average age of $57.0 \pm 12.4$ years. The mean hemodialysis vintage was $45.0 \pm 45.9$ months. Diabetic nephropathy was the leading cause of $\operatorname{ESRD}(\mathrm{n}=9)$.

Although all participants accomplished intradialytic exercise for 3 months, five patients dropped out from the 6-month work-up. The reasons of withdrawal included procedures such as transarterial chemoembolization $(n=1)$, transfers of dialysis units $(n=2)$, a death because of a brain haemorrhage $(n=1)$, and voluntary interrup$\operatorname{tion}(n=1)$. None of these reasons was related to exercise. Over the course of exercise, some patients complained of somatic leg pain symptoms (especially knee), cramps, tiredness, weakness, and oedema of lower extremities.

Table 1. Baseline characteristics of the participants $(n=22)$

\begin{tabular}{|c|c|}
\hline Variable & Value \\
\hline Sex, male:female & $9: 13$ \\
\hline Age, yr & $57.0 \pm 12.4$ \\
\hline Weight, kg & $57.6 \pm 11.77$ \\
\hline Body mass index, kg/m² & $24.1 \pm 4.02$ \\
\hline Duration of dialysis, mon & $45 \cdot 0 \pm 45 \cdot 9$ \\
\hline Smoking history & 7 \\
\hline \multicolumn{2}{|l|}{ Comorbidity } \\
\hline Diabetes & 12 \\
\hline Hypertension & 20 \\
\hline Congestive heart failure & 4 \\
\hline Ischemic heart disease & 6 \\
\hline Etc. & 5 \\
\hline \multicolumn{2}{|l|}{ Aetiology of ESRD } \\
\hline Diabetes & 9 \\
\hline Hypertension & 6 \\
\hline Glomerular disease & 2 \\
\hline Other causes & 5 \\
\hline Hemoglobin, g/dL & $10.0 \pm 1.3$ \\
\hline Urea, mg/dL & $61.6 \pm 17.6$ \\
\hline Creatinine, mg/dL & $9.0 \pm 2.4$ \\
\hline Potassium, mEq/L & $4.8 \pm 0.8$ \\
\hline Sodium, mEq/L & $139.2 \pm 2.4$ \\
\hline Calcium, mg/dL & $8.3 \pm 0.4$ \\
\hline Phosphorus, mg/dL & $4.6 \pm 1.8$ \\
\hline Albumin, g/dL & $3.9 \pm 0.4$ \\
\hline
\end{tabular}

Values are presented as mean $\pm \mathrm{SD}$.

ESRD, end-stage renal disease. 


\section{Effect on physical function}

As shown in Table 2, there were significant improvements in back muscle power, forward trunk flexibility, backward trunk flexibility, vertical jump, elbow flexion, sit-to-stand test, and 6-minute walk test $(p<0.01)$ over time. For grasp power, the mean value of month 6 was increased compared to values of month o and 3. However, these changes were not statistically significant. In addition, no significant differences were noted in arm and thigh circumferences after 6 months.

\section{Clinical parameters of dialysis and changes of labo- ratory results over 6 months}

The changes in blood pressure before, during, and after exercise are shown in Fig. 1. As the exercise progressed, the decrease in blood pressure during exercise was gradually improved, especially in systolic blood pressure(Fig. 2). Six patients never had IDH events during data collection period. For the rest of 16 patients, the IDH episodes from baseline to month 6 was significantly reduced $(p<$ o.05). However, there were no significant effects in the rest of parameters including dry weight, systolic blood pressure, diastolic blood pressure, and Kt/V (Table 3). At the end of the study, no significant changes were observed in hematological and biochemical variables after repeated measure analysis (data not shown).

\section{Depression}

In Table 4, BDI showed a lower score (8.73) at month 6 and a higher score (13.74) at month o with statistically significant improvement $(p<0.01)$.

\section{Quality of life}

Table 5 showed no significant increase in each domain, except for bodily pain (BP). Measured values of BP were 68.6 for month 6 and 78.3 for month $o(p<0.05)$. This result indicates that the value of $\mathrm{BP}$ decreased as the time passed.

\section{DISCUSSION}

Following a 6-month intradialytic exercise study, there have been several changes when compared to the baseline data. The equipment used for exercise was costeffective, space-saving, and feasible to administer. Additionally, cardiac decompensation was prevented by performing exercise within first half hours of hemodialysis $[11,12]$. No common dangerous adverse events caused by exercise including musculoskeletal injuries and serious cardiovascular complications such as ischemic heart disease and arrhythmia occurred. Only mild adverse symptoms and IDH were observed.

Especially, in dialysis population, not just diminished muscle mass and altered muscle quality but also decreased physical functioning can lead to falling accidents. Moreover, sedentary life style with low physical activity was associated with higher cardiovascular mortality [13]. These features were substantially related to increased morbidity and mortality risk [14,15]. Therefore, the improvement of physical function could be achieved by the exercise, which promotes increased blood flow to peripheral tissues and enhances perfusion of muscle

Table 2. Effects of 6-month intradialytic exercise on physical function

\begin{tabular}{lcccc}
\hline Variable & Month o & Month 3 & Month 6 & $p$ value \\
\hline Grasp power, kg & $22.1 \pm 6.2$ & $22.2 \pm 6.8$ & $23.3 \pm 6.8$ & 0.126 \\
Back muscle power, kg & $44.5 \pm 22.1$ & $48.5 \pm 21.5$ & $56.9 \pm 25.7$ & 0.000 \\
Forward trunk flexibility, cm & $5.0 \pm 12.6$ & $6.1 \pm 10.3$ & $10.8 \pm 9.0$ & 0.008 \\
Backward trunk flexibility, cm & $20.6 \pm 10.6$ & $21.9 \pm 10.5$ & $24.0 \pm 11.2$ & 0.005 \\
Vertical jump, cm & $13.9 \pm 7.5$ & $16.2 \pm 8.5$ & $18.8 \pm 8.7$ & 0.000 \\
Elbow flexion & $26.0 \pm 9.3$ & $29.4 \pm 6.6$ & $39.5 \pm 9.6$ & 0.000 \\
Sit-to-stand & $18.5 \pm 6.4$ & $21.9 \pm 8.0$ & $29.9 \pm 10.3$ & 0.000 \\
Arm circumference, cm & $27.5 \pm 3.3$ & $28.5 \pm 2.8$ & $28.3 \pm 3.3$ & 0.307 \\
Thigh circumference, cm & $39.7 \pm 7.2$ & $41.4 \pm 5.7$ & $39.8 \pm 5.1$ & 0.946 \\
6-Minute walk test, $\mathrm{m}$ & $377.7 \pm 93.1$ & $401.4 \pm 96.1$ & $425.8 \pm 92.8$ & 0.004 \\
\hline
\end{tabular}

Values are presented as mean \pm SD. Repeated measurement of analysis of variance. $p<0.05$. 

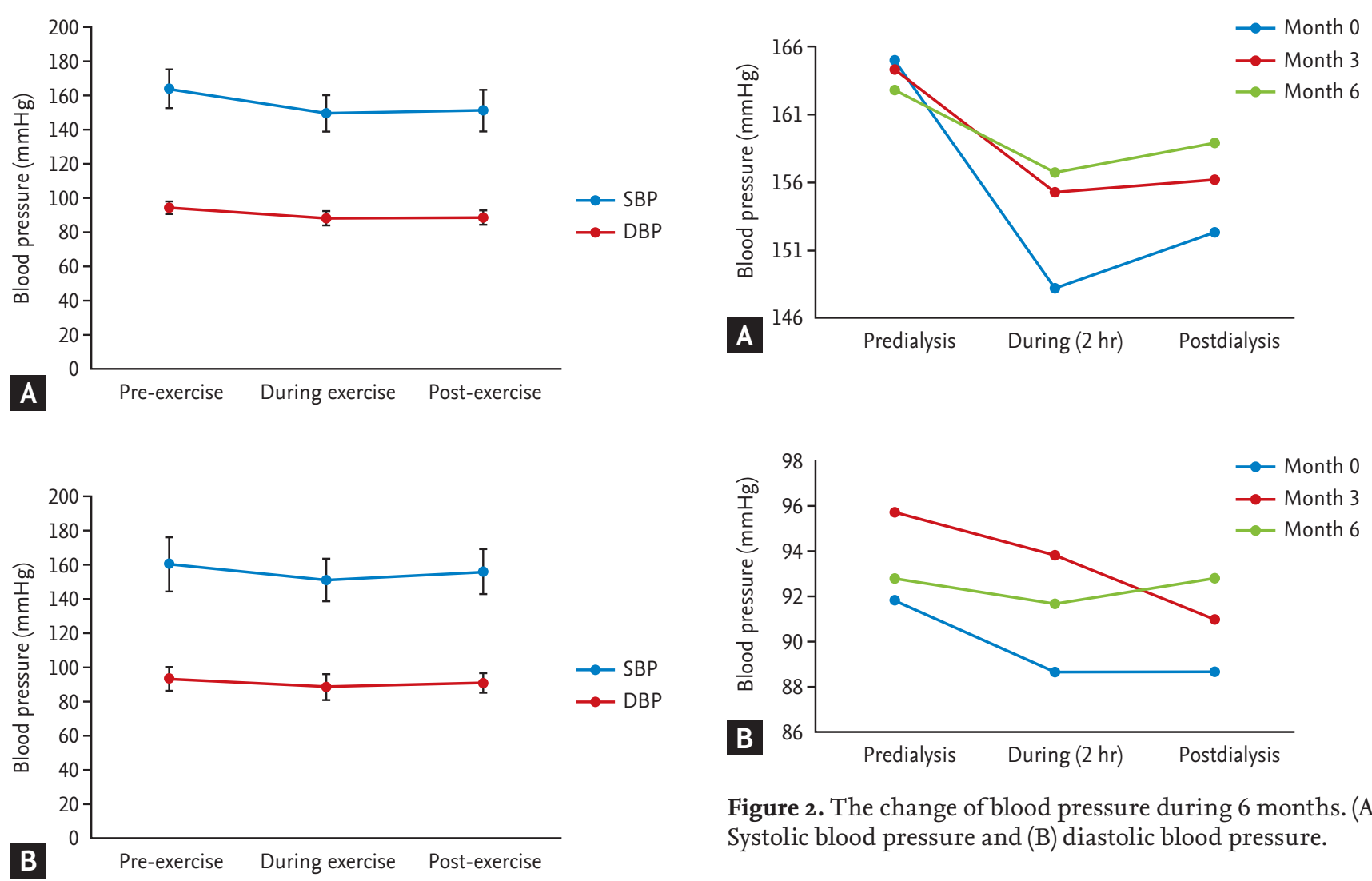

Figure 2. The change of blood pressure during 6 months. (A) Systolic blood pressure and (B) diastolic blood pressure.

Figure 1. The pattern of blood pressure before, during and after exercise. (A) Intradialytic hypotension (+) group $(n=16)$ and $(B)$ intradialytic hypotension $(-)$ group $(n=6)$. Values are expressed as mean \pm SD. SBP, systolic blood pressure; DBP, diastolic blood pressure.

cells [9].

In the present study, anaerobic exercise was used to induce muscle contractions and to increase muscle strength and the size of skeletal muscles [16]. In addition, the control of exercise intensity was carried out individ-

ually because a significant overload and progression of exercise is crucial for hypertrophic adaptation [17].

Based on our results, intradialytic exercise led to significant enhancement of back muscle power, elbow flexion test, sit-to-stand test, trunk flexibility test (forward and backward), vertical jump, and 6-minute walk test in terms of physical function. The results of measurements and tests reflected muscle strength, muscle endurance, lower extremity function, flexibility, balance,

Table 3. Dialysis related results at baseline and during follow-up

\begin{tabular}{lcccc}
\hline Variable & Month o & Month 3 & Month 6 & $p$ value \\
\hline DW, kg & $57.60 \pm 11.77$ & $57.92 \pm 11.73$ & $57.62 \pm 11.52$ & 0.960 \\
SBP, $\mathrm{mmHg}$ & $160.53 \pm 14.33$ & $162.11 \pm 16.19$ & $158.95 \pm 11.97$ & 0.578 \\
DBP, mmHg & $91.05 \pm 7.37$ & $93.16 \pm 8.85$ & $91.05 \pm 7.37$ & 1.000 \\
kt/V & $1.51 \pm 0.33$ & $1.65 \pm 0.39$ & $1.53 \pm 0.29$ & 0.823 \\
IDH (patient) & 16 & 10 & 6 & 0.019 \\
IDH (episode) & $2.22 \pm 2.83$ & $1.66 \pm 2.65$ & $1.06 \pm 1.92$ & \\
\hline
\end{tabular}

Values are presented as mean \pm SD. Repeated measurement of analysis of variance. $p<0.05$.

DW, dry weight; SBP, systolic blood pressure; DBP, diastolic blood pressure; IDH, intradialytic hypotension.

${ }^{a}$ Total numbers and not mean values of patients are represented. 
coordination, and cardiorespiratory endurance [5,18]. It is therefore tempting to speculate that the exercise was beneficial for improving functional capacity, muscle power, endurance, fatigability, and cardiorespiratory function in dialysis patients [5,6,19].

On the other hand, HGS and circumferences of arm and thigh reflecting muscle mass did not show any statistical improvement after exercise. Vogt et al. [20] reported that HGS cut off values that predict mortality were $22.5 \mathrm{~kg}$ for men and $7 \mathrm{~kg}$ for women. Those values were also associated with nutritional status and muscle function [21]. Although there was no statistically significant increase of HGS, the baseline value of $22.1 \mathrm{~kg}$ increased to $23.3 \mathrm{~kg}$ in 6 months. Moreover, the other

Table 4. BDI data for depression

\begin{tabular}{lcccc}
\hline Variable & Month o & Month 3 & Month 6 & p value \\
\hline BDI & $13.4 \pm 6.7$ & $10.3 \pm 4.2$ & $8.7 \pm 4.2$ & 0.004 \\
Clinical diagnosis & & & & - \\
$\quad$ No depression & 15 & 18 & 15 & \\
Mild depression & 4 & 1 & 1 & \\
$\quad \begin{array}{l}\text { Moderate } \\
\text { depression }\end{array}$ & 3 & 1 & 0 & \\
\hline
\end{tabular}

Values are presented as mean \pm SD. For clinical diagnosis, total numbers and not mean values of patients are represented. Repeated measurement of analysis of variance. $p<$ 0.05 .

BDI, Beck Depression Inventory. study reported that muscle strength association with mortality was greater than muscle mass association in dialysis patients [22].

Most patients with loss of renal function eventually develop anaemia, dysfunction of bone metabolism, and immunologic compromise [11] as they maintain hemodialysis treatment. Consequently, many researches have been done on various biochemical indices looking advancements. The results were controversial. Some studies have shown no changes after intradialytic exercise in oxidative stress, inflammation, and calcium content $[11,23]$. On the contrary, other studies demonstrated post-exercise improvement in dialysis adequacy, Kt/V [9], blood pressure [11], anemia [24], inflammation [25], and phosphorus clearance [26]. However, our findings on laboratory, and dialysis related parameters did not show any enhancement after 6 months of exercise, except for IDH.

It is known that IDH in hemodialysis patient occurs through various and complex pathophysiology; sudden reduction of circulating volume owing to ultrafiltration, decreased extracellular osmolality associated with sodium removal, overproduction of nitric oxide, decreased catecholamine and rennin release, and cardiac underfilling and impaired cardiovascular compensatory mechanism [27]. Also, patients with ESRD on HD commonly have autonomic nervous system dysfunction by alteration of decreased baroreceptor sensitivity, or the

Table 5. Quality of life measurements by Short Form-36 health survey

\begin{tabular}{lcccc}
\hline Variable & Month o & Month 3 & Month 6 & $p$ value \\
\hline PF & $62.1 \pm 21.1$ & $64.7 \pm 19.9$ & $59.7 \pm 18.2$ & 0.608 \\
RP & $55.2 \pm 24.8$ & $62.2 \pm 23.9$ & $58.5 \pm 25.0$ & 0.694 \\
BP & $78.4 \pm 20.4$ & $75.4 \pm 17.7$ & $68.7 \pm 18.3$ & 0.014 \\
GH & $41.8 \pm 13.5$ & $41.8 \pm 14.6$ & $37.1 \pm 12.9$ & 0.268 \\
VT & $45.2 \pm 23.3$ & $54.1 \pm 22.0$ & $49.3 \pm 18.7$ & 0.494 \\
SF & $58.8 \pm 24.9$ & $75.7 \pm 21.9$ & $67.7 \pm 23.4$ & 0.157 \\
RE & $53.9 \pm 27.7$ & $68.6 \pm 27.7$ & $65.7 \pm 24.8$ & 0.141 \\
MH & $65.9 \pm 17.2$ & $74.4 \pm 17.5$ & $71.2 \pm 14.6$ & 0.360 \\
PCS & $59.4 \pm 11.4$ & $61.0 \pm 13.9$ & $56.0 \pm 12.8$ & 0.304 \\
MCS & $56.0 \pm 14.7$ & $68.2 \pm 17.4$ & $63.5 \pm 16.0$ & 0.062 \\
Total score & $57.7 \pm 11.4$ & $64.6 \pm 14.4$ & $59.7 \pm 13.6$ & 0.526 \\
\hline
\end{tabular}

Values are presented as mean \pm SD. Repeated measurement of analysis of variance for Short Form- 36 survey. $p<0.05$. PF, physical functioning; RP, role-physical; BP, bodily pain; GH, general health; VT, vitality; SF, social functioning; RE, roleemotional; MH, mental health; PCS, physical component summary; MCS, mental component summary. 
parasympathetic or sympathetic pathways [28].

The blood pressure rises when ordinary people exercise with a bicycle ergometer. Therefore, it was used as a tool for predicting the risks of future hypertension in patients with pre-hypertension [29]. However, there were cases that had reduced blood pressure [30] or no blood pressure changes [31] after the exercise. Also, the blood pressure decreased [32] or did not alter [33] after anaerobic elastic band exercise.

According to Fotbolcu et al. [28], ESRD patients with IDH have inadequate sympathetic activity of the cardiovascular system and impaired left ventricular systolic function during treadmill exercise compared to patients without IDH. Although the increase in blood pressure was less, blood pressure of those IDH patients were increased after initiation of exercise compared to preexercise blood pressure.

However, as shown in Fig. 1 of our study, IDH (+) and IDH (-) groups displayed decreased blood pressure during exercise compared to the pre-exercise blood pressure. Those different outcomes may be attributed to the inclusion of patients with anti-hypertensive medications or cardiovascular diseases and the intradialytic exercise.

In Fig. 2, the degree of decrease in blood pressure after dialysis was alleviated when the exercise was performed during dialysis for several months. This suggests that intradialytic exercise was helpful for the improvement of the IDH.

IDH can result in the microcirculatory and microcardiac ischemia, which subsequently decreases cardiac output and increases mortality [34]. Thus, we can presume that IDH amelioration after exercise is useful in decreasing mortality and is associated with positive effects on blood pressure control. Further studies on the mechanism of alleviated IDH may be needed.

According to Carney et al. [35], hemodialysis patients have a similar degree of depression and anxiety compared to psychiatric patients. Besides, severe depression can lower patient's mood and decrease his or her interest in all activities [36]. This symptom of depression might be relieved after exercise through increased levels of neurotransmitters such as serotonin and endorphins [19]. Additionally, it was demonstrated that most severely depressed patients had the greatest benefits [37].

In current study, the widely used scale of depression,
BDI score, got better with a statistical significance after exercise training, taking into account that intradialytic exercise is advantageous for psychological health status after all.

Finally, $\mathrm{SF}_{3} 6$ health survey is a reliable and valid appraising tool of QOL, which is extensively used for hemodialysis patients [38]. There have been several studies using $\mathrm{SF}_{3} 6$ for the effect of intradialytic exercise on QOL. Some studies showed significantly higher score of physical component summary compared to the score of mental component summary $[7,12]$. Improvements in other domains have been also shown [11]. However, our findings about the QOL revealed a significant increase in the area of bodily pain score only.

Unlike improved physical function parameters after exercise prescription, the physical functioning score of $\mathrm{SF}_{3} 6$ survey did not show significant enhancement. It can be explained with a similar case which compared self-reported health-related QOL with the objective of exercise performance in patients with congenital heart disease [39]. Even though the subjects of study were different, there was severe overestimation of physical functioning in most patients when compared with actual exercise test results. Self-estimated physical functioning poorly predicted actual exercise capacity.

Also, because self-reported physical functioning score in the QOL survey assesses level of difficulty performing activities of daily living, instrumental activities of daily living, and more strenuous activities, the gap between objective physical function parameters and subjective physical functioning score in $\mathrm{SF}_{3} 6$ survey can exist [40].

This study has many limitations. First, because the study was carried out in one dialysis centre, a small number of participants were recruited. In addition, there was no contemporaneous control group. Second, the patients had to work out with limited resources in a fixed time. The arm with arterio-venous fistula was excluded from exercise, which might have had influence on our results. Third, patients with relatively high average activity levels were chosen for the study. It is hard to generalize the results to all dialysis patients because of the selection bias. For last, we did not take any consideration about maintaining same dialysis mode, medication, or other dialysis-related conditions. Moreover, five patients dropped out before the 6th month of the study which might have also have affected the study results. 
As per our literature review, this was the first study to evaluate the effect of intradialytic exercise on IDH; this is a major strength of the study. Furthermore, we could identify diverse influences of intradialytic exercise.

In summary, combined aerobic and anaerobic exercise training on dialysis days was found to have positive effects on physical health status. Besides, diminished IDH events and bodily pain after intradialytic exercise resulted in improved depression. This improvement is theoretically beneficial for morbidity and mortality reduction among hemodialysis patients. Therefore, the findings of the current study may provide an appropriate guidance for encouraging exercise by nephrologists. Because exercise method was adjusted for the typical outpatients who have a relatively good physical function, the novel exercise programs for less healthy patients will have to be researched continually. In addition, we recommend for that more clinical guidelines to be revised in order to encourage clinicians to actively implement intradialytic exercise programs.

\section{KEY MESSAGE}

1. Combined aerobic and anaerobic exercise training during dialysis was found to be effective on physical health status, intradialytic hypotension, and depression in terms of mental health.

2. The findings of the current study may provide an appropriate guidance for encouraging exercise by nephrologists.

\section{Conflict of interest}

No potential conflict of interest relevant to this article was reported.

\section{Acknowledgments}

We are grateful to the patients for their invaluable cooperation. We appreciate all medical and nursing staff of dialysis unit from Hallym University Kangdong Sacred Heart Hospital. Jieun Oh is supported by the Baxter grant from the Korean Society of Nephrology, Republic of Korea.

\section{REFERENCES}

1. Saran R, Li Y, Robinson B, et al. US Renal Data System 2015 Annual Data Report: epidemiology of kidney disease in the United States. Am J Kidney Dis 2016;67(3 Suppl 1):Svii, $\mathrm{S}_{1}-\mathrm{S}_{305}$.

2. K/DOQI Workgroup. K/DOQI clinical practice guidelines for cardiovascular disease in dialysis patients. Am J Kidney Dis 2005;45(4 Suppl 3):S1-S153.

3. Fouque D, Vennegoor M, ter Wee P, et al. EBPG guideline on nutrition. Nephrol Dial Transplant 2007;22 Suppl 2:ii45-ii87.

4. Cano NJ, Fouque D, Roth $\mathrm{H}$, et al. Intradialytic parenteral nutrition does not improve survival in malnourished hemodialysis patients: a 2-year multicenter, prospective, randomized study. J Am Soc Nephrol 2007;18:2583-2591.

5. Kono K, Nishida Y, Moriyama Y, Yabe H, Taoka M, Sato $\mathrm{T}$. Investigation of factors affecting the six-minute walk test results in hemodialysis patients. Ther Apher Dial 2014;18:623-627.

6. Heiwe S, Jacobson SH. Exercise training in adults with CKD: a systematic review and meta-analysis. Am J Kidney Dis 2014;64:383-393.

7. Sheng K, Zhang P, Chen L, Cheng J, Wu C, Chen J. Intradialytic exercise in hemodialysis patients: a systematic review and meta-analysis. Am J Nephrol 2014;40:478-490.

8. Silva LC, Marinho PE. Knowledge among nephrologists about the importance of exercise in the intradialytic period. J Phys Ther Sci 2015;27:2991-2994.

9. Parsons TL, Toffelmire EB, King-VanVlack CE. Exercise training during hemodialysis improves dialysis efficacy and physical performance. Arch Phys Med Rehabil 2006;87:680-687.

10. Bullani R, El-Housseini Y, Giordano F, et al. Effect of intradialytic resistance band exercise on physical function in patients on maintenance hemodialysis: a pilot study. $J$ Ren Nutr 2011;21:61-65.

11. Musavian AS, Soleimani A, Masoudi Alavi N, Baseri A, Savari F. Comparing the effects of active and passive intradialytic pedaling exercises on dialysis efficacy, electrolytes, hemoglobin, hematocrit, blood pressure and healthrelated quality of life. Nurs Midwifery Stud 2015;4:e25922.

12. Jung TD, Park SH. Intradialytic exercise programs for hemodialysis patients. Chonnam Med J 2011;47:61-65.

13. O'Hare AM, Tawney K, Bacchetti P, Johansen KL. Decreased survival among sedentary patients undergoing 
dialysis: results from the dialysis morbidity and mortality study wave 2. Am J Kidney Dis 2003;41:447-454.

14. Sietsema KE, Amato A, Adler SG, Brass EP. Exercise capacity as a predictor of survival among ambulatory patients with end-stage renal disease. Kidney Int 2004;65:719-724.

15. Kurella Tamura M, Covinsky KE, Chertow GM, Yaffe K, Landefeld CS, McCulloch CE. Functional status of elderly adults before and after initiation of dialysis. $\mathrm{N}$ Engl J Med 2009;361:1539-1547.

16. Gianola S, Pecoraro V, Lambiase S, Gatti R, Banfi G, Moja L. Efficacy of muscle exercise in patients with muscular dystrophy: a systematic review showing a missed opportunity to improve outcomes. PLoS One 2013;8:e65414.

17. Kraemer WJ, Adams K, Cafarelli E, et al. American College of Sports Medicine position stand: progression models in resistance training for healthy adults. Med Sci Sports Exerc 2002;34:364-380.

18. Torino C, Manfredini F, Bolignano D, et al. Physical performance and clinical outcomes in dialysis patients: a secondary analysis of the EXCITE trial. Kidney Blood Press Res 2014;39:205-211.

19. Esteve Simo V, Junque A, Fulquet M, et al. Complete lowintensity endurance training programme in haemodialysis patients: improving the care of renal patients. Nephron Clin Pract 2014;128:387-393.

20. Vogt BP, Borges MC, Goes CR, Caramori JC. Handgrip strength is an independent predictor of all-cause mortality in maintenance dialysis patients. Clin Nutr 2016;35:1429-1433.

21. Norman K, Stobaus N, Gonzalez MC, Schulzke JD, Pirlich M. Hand grip strength: outcome predictor and marker of nutritional status. Clin Nutr 2011;30:135-142.

22. Isoyama N, Qureshi AR, Avesani CM, et al. Comparative associations of muscle mass and muscle strength with mortality in dialysis patients. Clin J Am Soc Nephrol 2014;9:1720-1728.

23. Esgalhado M, Stockler-Pinto MB, de Franca Cardozo LF, Costa C, Barboza JE, Mafra D. Effect of acute intradialytic strength physical exercise on oxidative stress and inflammatory responses in hemodialysis patients. Kidney Res Clin Pract 2015;34:35-40.

24. Falahi MJ, Shahidi S, Farajzadegan Z. The effect of intradialytic exercise on dialysis efficacy, serum phosphate, hemoglobin and blood pressure control and comparison between two exercise programs in hemodialysis patients. J Isfahan Med Sch 2008;26:152-161.
25. Castaneda C, Gordon PL, Parker RC, Uhlin KL, Roubenoff R, Levey AS. Resistance training to reduce the malnutrition-inflammation complex syndrome of chronic kidney disease. Am J Kidney Dis 2004;43:607-616.

26. Vaithilingam I, Polkinghorne KR, Atkins RC, Kerr PG. Time and exercise improve phosphate removal in hemodialysis patients. Am J Kidney Dis 2004;43:85-89.

27. Sulowicz W, Radziszewski A. Pathogenesis and treatment of dialysis hypotension. Kidney Int 2006;70:S36-S39.

28. Fotbolcu H, Duman D, Ecder SA, et al. Attenuated cardiovascular response to sympathetic system activation during exercise in patients with dialysis-induced hypotension. Am J Nephrol 2011;33:491-498.

29. Miyai N, Arita M, Miyashita K, Morioka I, Shiraishi T, Nishio I. Blood pressure response to heart rate during exercise test and risk of future hypertension. Hypertension 2002;39:761-766.

30. Milatz F, Ketelhut S, Ketelhut S, Ketelhut RG. Favorable effect of aerobic exercise on arterial pressure and aortic pulse wave velocity during stress testing. Vasa 2015;44:271276.

31. Siegelova J, Havelkova A, Dusek J, et al. 3D.o6: blood pressure variability at rest and during exercise in healthy men: seven day ambulatory blood pressure monitoring. J Hypertens 2015;33 Suppl 1:e42.

32. Park J. Effects of 24-week resistance exercise training on carotid peak systolic and end diastolic flow velocity in healthy older adults. J Phys Ther Sci 2016;28:2793-2797.

33. Yasuda T, Fukumura K, Tomaru T, Nakajima T. Thigh muscle size and vascular function after blood flow-restricted elastic band training in older women. Oncotarget 2016;7:33595-33607.

34. Flythe JE, Xue H, Lynch KE, Curhan GC, Brunelli SM. Association of mortality risk with various definitions of intradialytic hypotension. J Am Soc Nephrol 2015;26:724734 .

35. Carney RM, McKevitt PM, Goldberg AP, Hagberg J, Delmez JA, Harter HR. Psychological effects of exercise training in hemodialysis patients. Nephron 1983;33:179181.

36. Kouidi E, Karagiannis V, Grekas D, et al. Depression, heart rate variability, and exercise training in dialysis patients. Eur J Cardiovasc Prev Rehabil 2010;17:160-167.

37. Kouidi E, Iacovides A, Iordanidis $\mathrm{P}$, et al. Exercise renal rehabilitation program: psychosocial effects. Nephron 1997;77:152-158. 
38. Mingardi G, Cornalba L, Cortinovis E, Ruggiata R, Mosconi P, Apolone G. Health-related quality of life in dialysis patients: a report from an Italian study using the SF-36 Health Survey. DIA-QOL Group. Nephrol Dial Transplant 1999;14:1503-1510.

39. Gratz A, Hess J, Hager A. Self-estimated physical func- tioning poorly predicts actual exercise capacity in adolescents and adults with congenital heart disease. Eur Heart J 2009;30:497-504.

40. Painter P. Physical functioning in end-stage renal disease patients: update 2005. Hemodial Int 2005;9:218-235. 